\title{
The role of duloxetine in the treatment of anxiety disorders
}

\author{
Domenico De Berardis 1,2,3 \\ Nicola Serroni ${ }^{2}$ \\ Alessandro Carano ${ }^{1,4}$ \\ Marco Scali ${ }^{1,4}$ \\ Alessandro Valchera ${ }^{5}$ \\ Daniela Campanella ${ }^{1,2}$ \\ Alessandro D'Albenzio' \\ Berardo Di Giuseppe ${ }^{2}$ \\ Francesco Saverio \\ Moschetta ${ }^{2}$ \\ Rosa Maria Salerno' \\ Filippo Maria Ferro' \\ 'Department of Oncology and \\ Neurosciences, Institute of Psychiatry, \\ "G. D’Annunzio" University of Chieti, \\ Italy; ${ }^{2}$ National Health Service, \\ Department of Mental Health, SPDC \\ Teramo, Italy; ${ }^{3} \mathrm{ITAB}$, Institute for \\ Advanced Biomedical Technologies, \\ “G. D’Annunzio" University \\ Foundation, Chieti, Italy; ${ }^{4}$ National \\ Health Service, Department of Mental \\ Health, ASUR Marche 8, Civitanova \\ Marche, Italy; ${ }^{5}$ Division of Psychiatry, \\ "S. Giuseppe" Clinic, Ascoli Piceno, Italy
}

Correspondence: Domenico De Berardis Department of Oncology and Neurosciences, Institute of Psychiatry, University "G. D'Annunzio" of Chieti, Palazzina SE.BI. - Scuole di Specializzazione, via dei Vestini, 3I - 660I3 Chieti, Italy Tel $+39087 \mid 3556750$

Fax +3908713556721

Email dodebera@aliceposta.it

\begin{abstract}
Anxiety disorders (ADs) are the most common type of psychiatric disorders, with a mean incidence of $18.1 \%$ and a lifetime prevalence of $28.8 \%$. Pharmacologic options studied for treating ADs may include benzodiazepines, tricyclic antidepressants (TCA), selective serotonin reuptake inhibitors (SSRIs), noradrenergic and specific serotonergic drug (NaSSA) and dual-reuptake inhibitors of serotonin and norepinephrine (SNRIs). In this context, the development of SNRIs (venlafaxine and duloxetine) has been particularly useful. As a dual-acting intervention that targets two neurotransmitter systems, these medications would appePar promising for the treatment of ADs. The purpose of this review was to elucidate current facts and views about the role of duloxetine in the treatment of ADs. In February 2007, duloxetine was approved by FDA for the treatment of generalized anxiety disorder (GAD). The results of trials evaluating the use duloxetine in the treatment of GAD are supportive on its efficacy even if further studies on long-term use are needed. Apart from some interesting case reports, no large studies are, to date, present in literature about duloxetine and other ADs such as panic disorder, social anxiety disorder, obsessive-compulsive disorder and post-traumatic stress disorder. Therefore, the clinical efficacy and the relative good tolerability of duloxetine may be further investigated to widen the therapeutic spectrum of ADs.
\end{abstract}

Keywords: anxiety disorders, duloxetine, serotonin, noradrenaline, efficacy, tolerability

\section{Introduction}

Anxiety disorders (ADs) are the most common type of psychiatric disorders, with a mean incidence of $18.1 \%$ and a lifetime prevalence of $28.8 \%$ (Kessler et al 2005). ADs results in a great economic burden as it was estimated that they account for a $\$ 42.3$ billion annual cost in the United States, with over $50 \%$ of the total sum directed towards nonpsychiatric medical treatment costs (Greenberg et al 1999). Moreover, Wang and colleagues (2005), in the National Comorbidity Survey Replication, pointed out that only about $37 \%$ of patients with ADs utilize any form of health services, including psychiatric consultation (13\%), other mental health practitioners $(16 \%)$, or primary care physicians $(24.3 \%)$.

To date, it is well established that patients suffering of AD may also have a high comorbidity with mood disorders, with up to $90 \%$ of patients experiencing some kind of depressive disorder in their lifetime with these two disorders sharing some neurobiological mechanisms such as abnormalities in the 5HT1A receptor function (Nutt and Stein 2006).

Due the involvement of multiple neurobiological systems, several classes of medications have been widely used for the treatment of ADs. The main classes include benzodiazepines, tricyclic antidepressants (TCA), buspiron hydrochloride, selective serotonin reuptake inhibitors (SSRIs), noradrenergic and specific serotonergic drug (NaSSA), and dual-reuptake inhibitors of serotonin and noradrenaline (SNRIs) (Goodman 2004; Gambi et al 2005; Shearer 2007; Sheehan and Sheehan 2007). It has been demonstrated that a novel agent acting on GABA neurotransmission, pregabalin, was effective in the treatment of generalized anxiety disorder (GAD) (Rickels et al 2005) 
and, in 2006, it was officially approved in European Union for the treatment of GAD. However, some of these drugs do not have a favorable profile in the long-term treatment of $\mathrm{AD}$. In fact, even if benzodiazepines can be useful for the immediate alleviation of anxiety, their effectiveness for long-term management has been questioned because of concerns about tolerance, dependency, and remission rates (Pollack 2001).

The SSRIs are preferred in the treatment of AD because of greater safety and tolerability an to date are licensed for the treatment of several AD including panic disorder (PD), GAD, obsessive-compulsive disorder (OCD), and social anxiety disorder (SAD) (Vaswani et al 2003). However, despite their favorable profile of side effects, these drugs may cause some adverse effects that may be problematic for some patients such as sexual dysfunction, risk of bleeding, hyponatremia, discontinuation symptoms, and increased bodyweight (Westenberg and Sandner, 2006; Williams et al 2006). In fact, it has been reported that patients with AD are more sensible to side effects of psychopharmacological treatment (Bandelow et al 2006). Furthermore, although patients with GAD respond to SSRIs, the minority of patients actually achieve remission with these treatments (Pollack 2001).

In this context, the development of SNRIs (venlafaxine and duloxetine) has been particularly useful. As a dual-acting intervention that targets two neurotransmitter systems, these medications would appear promising for the treatment of AD. The purpose of this review was to elucidate current facts and views about the role of the novel SNRI duloxetine in the treatment of $\mathrm{AD}$.

\section{Methods}

Searches of the Medline database from 1988 through January 2008 and the PsycInfo/Embase database from 1988 through January 2008 were conducted, restricted to the English language. The search term "duloxetine" was combined with "anxiety", "generalized anxiety disorder", "obsessivecompulsive disorder", "panic disorder", "phobia”, "social anxiety disorder", and "post-traumatic stress disorder" to identify relevant original research and review articles. All citations were screened, and the full texts of peer-reviewed journal articles considered relevant to the purposes of the review were obtained. Bibliographies were scanned to locate additional relevant publications, even those older than 1988.

\section{Characteristics of duloxetine}

Duloxetine [LY248686, (+)-N-methyl-3-(1-naphthalenyloxy)2-thiophenepropanamine], a relatively new antidepressant drug, was discovered in 1988 by Wong and colleagues and released in August 2004. Duloxetine is an inhibitor of the reuptake of serotonin (5-hyroxytryptamine; 5HT) and noradrenaline (NA), both in vitro and in vivo (Wong et al 1993). Duloxetine has demonstrated a relatively evenly balanced and potent inhibition of both the 5HT and NE reuptake at the transport sites and a weak effect on dopamine reuptake in both in vitro and in vivo studies (Pitsikas 2000; Wong 1998; Wong and Bymaster 2002). Duloxetine lacks significant affinity for muscarinic, histamine $\mathrm{H} 1$, alpha1-adrenergic, dopamine D2, 5HT1A, 5HT1B, 5HT1D, 5HT2A, 5HT2C, GABA, glutamate and opioid receptors (Wong et al 1993; Bymaster et al 2001). Moreover, duloxetine does not exert its action through the inhibition of monoamine oxidase (MAO).

Duloxetine is rapidly absorbed following oral administration. Absorption of duloxetine begins two hours after oral administration, and the maximum concentration in plasma (C-max) is achieved in approximately six hours (Bymaster et al 2003). The estimated volume of distribution is $1640 \mathrm{~L}$, although there was large variability among subjects (Sharma et al 2000). Duloxetine is highly protein bound (96\%), primarily to albumin or $\alpha 1$-acidglycoprotein (Bymaster et al 2003). Duloxetine undergoes extensive metabolism, but the major circulating metabolites have not been shown to contribute significantly to the pharmacologic activity of duloxetine itself (Westanmo et al 2005). It is metabolized primarily by cytochrome P-450 (CYP) isoenzymes 2D6 and 1A2 (Bymaster et al 2003). Duloxetine has a mean plasma half-life of 12 hours (Sharma et al 2000).

Duloxetine was approved by the US Food and Drug Administration (FDA) in August 2004 for the treatment of major depressive disorder (MDD). It has been suggested that, due to a more balanced inhibition of 5-HT and NE reuptake, duloxetine can provide enhanced benefits in relieving depression. It has also been hypothesized that duloxetine has less affinity for other receptors (eg, muscarinic) that are held responsible for the numerous adverse events of other antidepressants. Several studies have established the safety and efficacy of duloxetine in the treatment of MDD (Detke et al 2002; Nemeroff et al 2002; Lewis-Fernandez et al 2006; Shelton et al 2007). For an exhaustive review of efficacy and tolerability of duloxetine in MDD see Frampton and Plosker (2007).

Moreover, as duloxetine enhances urethral striated sphincter activity and increases bladder capacity in an in vivo cat bladder irritation model, this SNRI seems to be efficacious in the treatment of stress urinary incontinence (Moore 2004). Duloxetine has not been approved for stress 
urinary incontinence in the USA, but has been approved for stress urinary incontinence in the European Union since August 2004.

In September 2004 duloxetine was approved for the management of diabetic peripheral neuropathic pain. Even if the exact mechanism of central pain inhibition of duloxetine is, to date, not well known, it is thought to be related to potentiation of adrenergic and serotoninergic activity in the central nervous system (Wernicke et al 2006).

\section{Rationale for duloxetine treatment of ADs}

It has been widely demonstrated that MDD is associated with altered regulation of both 5-HT and NA neurotransmission and that acting on these two systems both separately or simultaneously may lead to therapeutic effects. Moreover, it is also well known that clinical observations demonstrate a significant degree of overlap in symptoms associated with MDD and ADs (Baldwin et al 2002). Although the precise mechanisms underlying the pathogenesis of anxiety remain unclear, a lot of evidences support decreased serotonergic function in both depression and anxiety.

Studies conducted on patients suffering of ADs have pointed out that a dysregulation of 5-HT neurotransmission such as low 5-HT turnover rate, is believed to play a role in the manifestation of symptoms of anxiety (Graeff et al 1997; Wrase et al 2006). Additionally, there have been reports of reduced serotonin transporter (SERT) binding in patients with GAD (Iny et al 1994). Consistent with these notions, antidepressants that enhance serotonergic neurotransmission, such as the SSRIs, have been found to be effective in treating some ADs, as above specified. Moreover, several genetic studies have demonstrated associations between certain polymorphisms in the 5-HT gene and anxiety traits as well as GAD (Lesch et al 1996; Ohara et al 1999). In addition, the short allele of the 5-HT gene linked polymorphic region has been associated with a greater activation of the amygdala in response to fearful face stimuli in healthy subjects (Hariri et al 2002).

The role of NA in anxiety is less clear, but many lines of evidence support the hypothesis that NA neurotransmission is involved in anxiety (Morilak et al 2005). NA has been shown to modulate activity in regions of the brain that are involved in anxiety, such as the amygdala and the locus coeruleus (Van Bockstaele 2000). In addition, increases in 3-methoxy-4-hydrophenylglycol (MHPG; a metabolite of NA) and hypersecretion of NA in plasma and cerebrospinal fluid have been associated with anxiety states (Bremner et al 1996).
Clinical evidences, including results from studies that have evaluated the anxiolytic efficacy of agents that modulate noradrenergic neurotransmission, are consistent with the involvement of NA in ADs (Stahl et al 2005).

Also preclinical studies have suggested anxiolytic effects for duloxetine. Using the mouse zero maze anxiety model, Troelsen and colleagues (2005) compared the effects of chronic administration of several antidepressants, including tricyclics, SSRIs, and SNRIs. Duloxetine demonstrated a significant anxiolytic effect via reduction in all of the animal behaviors associated with anxiety. The chronic duloxetine treatment regimen also resulted in a considerable reduction in serotonin transporters (SERT) density in the cortex after duloxetine whereas citalopram, amitriptyline and reboxetine were not anxiolytic after chronic treatment and induced a small or no reduction in SERT density.

These findings were also confirmed by Mirza and colleagues (2007) who showed that duloxetine and fluoxetine both reduced SERT density in mice cortex and induced anxiolytic-like effects. Moreover, duloxetine also reduced noradrenaline transporter (NET) density in mice cortex, with increased anxiolytic-like effects.

Moreover, as it has been demonstrated that duloxetine may antagonize the p-chloroamphetamine-induced depletion of 5-HT in the rat brain (Wong et al 1993), preventing also the depletion of NE induced by 6-hydroxydopamine in mice hearts and in the hypothalamus of rats (Fuller et al 1994), this may account for its action on anxiety symptoms: in addition, using microdialysis, it has been shown that acute systemic duloxetine administration may cause a large increase in extracellular levels of 5-HT and NE especially in the hypothalamus and cortex (Engleman et al 1995; Kihara and Ikeda 1995). Kasamo and colleagues (1996) found that duloxetine inhibits the spontaneous firing of both the dorsal raphe 5-HT and the locus coeruleus NE neurons, indicating an increased availability of these neurotransmitters in the immediate vicinity of their somatodendritic receptors regulating their firing activity.

\section{The role of duloxetine in the treatment of GAD}

The course of GAD appears, for its own nature, to require longterm treatment (Rynn and Brawman-Mintzer 2004). With this consideration, SSRIs and selective serotonin and noradrenaline reuptake inhibitors (SNRIs) have become first-line treatment interventions (Pollack 2001). The SNRI venlafaxine has been approved for GAD by the FDA as was shown to be effective in double-blind clinical trials (Rickels et al 2000). 
Efficacy of duloxetine on anxiety symptoms, especially in the treatment of GAD, has become object of investigation starting from the observation that duloxetine was effective in reducing anxiety symptoms associated with MDD. Dunner and colleagues (2003) analyzed all the placebo-controlled studies of duloxetine in MDD but focused on four trials in which duloxetine was superior to placebo on the primary outcome measure of the Hamilton Depression Rating Scale (HAM-D) total score, with anxiety symptoms assessed using the HAM-D anxiety/somatization subfactor and the anxiety-psychic item (HAM-D Item 10). They concluded that duloxetine was effective in treating a broad spectrum of symptoms associated with depression, including mood, anxiety, and painful physical symptoms. In particular, they pointed out that duloxetine caused a rapid relief of anxiety symptoms associated with depression. More recently, Fava and colleagues (2007) investigated the efficacy and tolerability of duloxetine in the treatment of anxious versus nonanxious depression with anxious depression defined by a HAM-D Anxiety/Somatization Factor score $\geq 7$. They observed that duloxetine treatment was associated with a significantly greater reduction in total HAM-D scores and HAM-D Anxiety/Somatization Factor scores among patients with anxious depression with the latter showing a significantly shorter median time to response than patients with nonanxious depression. Moreover, the tolerability of duloxetine was comparable between groups.

In February 2007, also duloxetine has been approved by FDA for the treatment of GAD and the double-blind, placebocontrolled, large sample ( $>100$ patients) studies of duloxetine in the treatment of GAD are summarized in Table 1. Overall, all these studies demonstrated that duloxetine was more effective than placebo and, at least, equal effective than venlafaxine XR in the treatment of GAD. Moreover, Russell and colleagues (2007) reported the effectiveness of duloxetine in improving not only anxiety symptoms, but also pain severity and patient functioning in adults with GAD, who presented with clinically significant pain symptoms. Interestingly, the data of the study of Hartford and colleagues (2007), Koponen and colleagues (2007) and Rynn and colleagues (2008) were also analyzed globally in two studies. In the first, Endicott and colleagues (2007) pooled data of these three clinical studies to report the efficacy of duloxetine in reducing role impairments and in enhancing wellbeing in patients with GAD and found that duloxetine consistently reduced role functioning disabilities associated with GAD and enhanced patients' quality of life and well-being. In addition, summarizing efficacy of the same three clinical studies, Allgulander and colleagues (2007) concluded that, considering a sample of more than 1100 patients, duloxetine was efficacious for reducing anxiety severity and for increasing patients overall role functioning in GAD.

Moreover, Pollack and colleagues (2008), pooling data from three double-blind, placebo-controlled clinical trials that examined the efficacy of duloxetine in GAD (Hartford et al 2007; Koponen et al 2007; Rynn et al 2008), found that patients with GAD experience significant and substantial impairment in three domains of quality of life: global role functioning, subjective well-being, and perceived health. Using the criterion of improvement to community values, patients treated with duloxetine were more likely to achieve this outcome on each quality of life measure.

However, it should be considered that all of the clinical trials were for short-term (9-10 weeks) acute treatment and therefore longer trials are necessary to determine the extended response and maintenance of effect from duloxetine treatment in GAD. To date, there are no long-term studies that examined whether the clinical efficacy of venlafaxine in treating GAD is maintained for 6 months or longer. Moreover, as patients with GAD frequently have comorbid psychiatric and medical illnesses and these trials excluded significant psychiatric and medical comorbidity, results of these studies should be extended also to, for example, primary-care settings to adding evidences to the generalizability of result to general patient population.

\section{Role of duloxetine in the treatment of other ADs}

Despite growing evidences about a good efficacy of duloxetine in the treatment of GAD, there is a relative lacking of data of its potential efficacy in other ADs such as $\mathrm{PD}, \mathrm{SAD}, \mathrm{OCD}$, and post-traumatic stress disorder (PTSD). In fact, for these disorders, there are only case reports that have suggested a potential efficacy. Crippa and Zuardi (2006) reported a case of a 26-year-old woman with PD and agoraphobia successfully treated with duloxetine whereas Luis Blay and Black (2007) showed a good clinical response in a 38-year-old man with OCD with a successful 1-year follow-up. Recently Dell'Osso and colleagues (2008) reported the cases of four patients with treatment-resistant OCD who were switched from SSRIs to duloxetine and followed up for 12 weeks. At the endpoint, three out of four patients showed a clinically significant improvement of the OC symptoms ( $\geq 35 \%$ on the Y-BOCS score), whereas the clinical features of the fourth patient remained unchanged. 


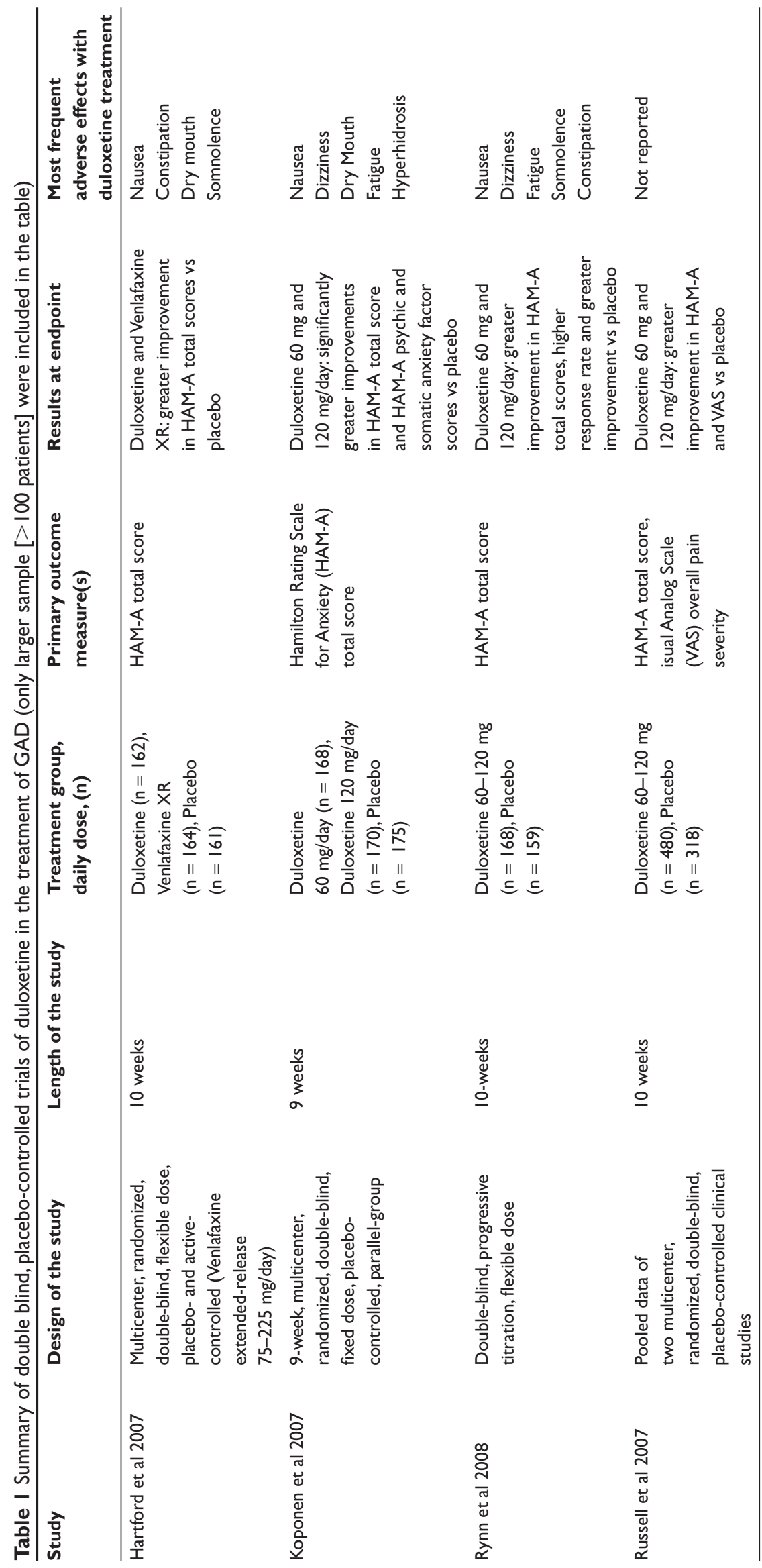


Again Crippa and colleagues (2007) pointed out that duloxetine treatment at $120 \mathrm{mg}$ /day was efficacious to reduce Brief Social Phobia Scale scores in two women with SAD.

Concerning PTSD, a case report showed an exacerbation of PTSD symptoms with use of duloxetine in a 53-year-old married Vietnam veteran, but this patient was also affected by bipolar disorder and this may potentially explain the finding along with sustained noradrenergic effects of this medication (Deneys and Ahearn 2007). On the other hand, Hanretta and Malek-Ahmadi (2007) reported a case of 38-year-old woman with treatment-resistant MDD and PTSD who was successfully treated with electroconvulsive therapy along with a duloxetine-olanzapine combination.

\section{Safety of duloxetine in the treatment of ADs}

The duloxetine treatment was generally well tolerated in the three studies of GAD where data on tolerability were available (Allgulander et al 2007; Endicott et al 2007). Nausea was the most frequent adverse effect reported by the patients in the studies of GAD. Treatment groups did not differ significantly in their overall study completion rates, which were $64.8 \%$ for duloxetine and $68.1 \%$ for placebo. The rate of discontinuation due to adverse event was $15.6 \%$ for the duloxetine group (mostly due to nausea and vomiting). No significant effect on weight was reported with the duloxetine treatment.

\section{Conclusions}

Duloxetine is a promising treatment for ADs that may be employed to obtain patient remission than merely response (getting the patient $50 \%$ better). The emerging data suggests that dual action agents may have greater efficacy in getting the patients with GAD to remission. However, the clinical efficacy of this drug will be more evident once will be more widely prescribed and used in the community by physicians. The results of trials evaluating the use duloxetine in the treatment of GAD are supportive on its efficacy on this disorder without severe adverse effects. Despite its clinical efficacy and FDA approval for the treatment of GAD, surprisingly, to date, the long term efficacy of duloxetine has not been yet investigated deeply and therefore further studies on long-term period are needed. Apart from some interesting case reports, no studies are, to date, present in literature about duloxetine and other ADs. Therefore, the clinical efficacy and the relative good tolerability of duloxetine in other ADs than GAD may be further investigated with double blind placebo-controlled studies in order to widen the therapeutic spectrum of ADs.

\section{Disclosure}

The authors report no conflicts of interest in this work.

\section{References}

Allgulander C, Hartford J, Russell J, et al. 2007. Pharmacotherapy of generalized anxiety disorder: results of duloxetine treatment from a pooled analysis of three clinical trials. Curr Med Res Opin, 23:1245-52.

Baldwin DS, Evans DL, Hirschfeld RM, et al. 2002. Can we distinguish anxiety from depression? Psychopharmacol Bull, 36:158-65.

Bandelow VB, Wolff-Menzler C, Wedekind D, et al. 2006. Anxiety disorders: long-term treatment and replapse prevention. MMW Fortschr Med, 148:31-4.

Bremner JD, Krystal JH, Southwick SM, et al. 1996. Noradrenergic mechanisms in stress and anxiety: II. Clinical studies. Synapse, 23:39-51.

Bymaster FP, Beedle EE, Findlay J, et al. 2003. Duloxetine (Cymbalta), a dual inhibitor of serotonin and norepinephrine reuptake. Bioorg Med Chem Lett, 13:4477-80.

Bymaster FP, Dreshfield-Ahmad LJ, Threlkeld PG, et al. 2001. Compare the affinity of duloxetine and venlafaxine for serotonin and norepinephrine transporters in vitro, human serotonin receptor subtypes, and other neuronal receptors. Neuropsychopharmacology, 25:871-80.

Crippa JA, Filho AS, Freitas MC, et al. 2007. Duloxetine in the treatment of social anxiety disorder. J Clin Psychopharmacol, 27:310.

Crippa JA, Zuardi AW. 2006. Duloxetine in the treatment of panic disorder. Int J Neuropsychopharmacol, 9:633-4.

Dell'osso B, Mundo E, Marazziti D, et al. 2008. Switching from serotonin reuptake inhibitors to duloxetine in patients with resistant obsessive compulsive disorder: a case series. J Psychopharmacol, 22:210-13.

Deneys ML, Ahearn EP. 2006. Exacerbation of PTSD symptoms with use of duloxetine. J Clin Psychiatry, 67:496-7.

Detke MJ, Lu Y, Goldstein DJ, et al. 2002. Duloxetine, $60 \mathrm{mg}$ once daily, for major depressive disorder: a randomized double-blind placebo controlled trial. J Clin Psychiatry, 63:308-15.

Dunner DL, Goldstein DJ, Mallinckrodt C, et al. 2003. Duloxetine in treatment of anxiety symptoms associated with depression. Depress Anxiety, 18:53-61.

Endicott J, Russell JM, Raskin J, et al. 2007. Duloxetine treatment for role functioning improvement in generalized anxiety disorder: three independent studies. J Clin Psychiatry, 68:518-24.

Engleman ES, Perry KW, Mayle DA, et al. 1995. Simultaneous increases of extracellular monoamines in microdyalisates from hypothalamus of conscious rats by duloxetine, a dual serotonin and norepinephrine uptake inhibitor. Neuropsychopharmacology, 12:287-95.

Fava M, Martinez JM, Greist J, et al. 2007. The efficacy and tolerability of duloxetine in the treatment of anxious versus nonanxious depression: a post-hoc analysis of an open-label outpatient study. Ann Clin Psychiatry, 19:187-95.

Frampton JE, Plosker GL. 2007. Duloxetine: a review of its use in the treatment of major depressive disorder. CNS Drugs, 21:581-609.

Fuller RW, Hemrick-Luecke SK, Snoddy HD. 1994. Effects of duloxetine, an antidepressant drug candidate, on concentrations of monoamines and their metabolites in rats and mice. J Pharmacol Exp Ther, 269:132-6.

Gambi F, De Berardis D, Campanella D, et al. 2005. Mirtazapine treatment of generalized anxiety disorder: a fixed dose, open label study. J Psychopharmacol, 19:483-7.

Goodman WK. 2004. Selecting pharmacotherapy for generalized anxiety disorder. J Clin Psychiatry, 65:8-13.

Graeff FG, Viana MB, Mora PO. 1997. Dual role of 5-HT in defense and anxiety. Neurosci Biobehav Rev, 21:791-9.

Greenberg PE, Sisitsky T, Kessler RC, et al. 1999. The economic burden of anxiety disorders in the 1990s. J Clin Psychiatry, 60:427-35.

Hanretta AT, Malek-Ahmadi P. 2007. Combined Use of ECT with Duloxetine and Olanzapine: A Case Report. J ECT, 22:139-41.

Hariri AR, Mattay VS, Tessitore A, et al. 2002. Serotonin transporter genetic variation and the response of the human amygdala. Science, 297:400-3. 
Hartford J, Kornstein S, Liebowitz M, et al. 2007. Duloxetine as an SNRI treatment for generalized anxiety disorder: results from a placebo and active-controlled trial. Int Clin Psychopharmacol, 22:167-74.

Iny LJ, Pecknold J, Suranyi-Cadotte BE, et al. 1994. Studies of a neurochemical link between depression, anxiety, and stress from $[3 \mathrm{H}]$ imipramine and $[3 \mathrm{H}]$ paroxetine binding on human platelets. Biol Psychiatry, 36:281-91.

Kasamo K, Blier P, de Montigny C. 1996. Blockade of the serotonin and norepinephrine uptake processes by duloxetine: In vitro and in vivo studies in the rat brain. $J$ Pharmacol Exp Ther, 277:278-86.

Kessler RC, Chiu WT, Demler O, et al. 2005. Prevalence, severity, and comorbidity of 12-month DSM-IV disorders in the National Comorbidity Survey Replication. Arch Gen Psychiatry, 62:617-27.

Kihara T, Ikeda M. 1995. Effects of duloxetine, a new serotonin and norepinephrine uptake inhibitor, on extracellular monoamine levels in rat frontal cortex. J Pharmacol Exp Ther, 272:177-83.

Koponen H, Allgulander C, Erickson J, et al. 2007. Efficacy of duloxetine for the treatment of generalized anxiety disorder: Implications for primary care physicians. Prim Care Companion J Clin Psychiatry, 9:100-7.

Lesch KP, Bengel D, Heils A, et al. 1996. Association of anxiety-related traits with a polymorphism in the serotonin transporter gene regulatory region. Science, 274:1527-31.

Lewis-Fernandez R, Blanco C, Mallinckrodt CH, et al. 2006. Duloxetine in the treatment of major depressive disorder: comparisons of safety and efficacy in U.S. Hispanic and majority Caucasian patients. $J$ Clin Psychiatry, 67:1379-90.

Luis Blay S, Black DW. 2007. A case of obsessive-compulsive disorder responding to duloxetine. Prim Care Companion J Clin Psychiatry, 9:234-5.

Mirza NR, Nielsen EØ, Troelsen KB. 2007. Serotonin transporter density and anxiolytic-like effects of antidepressants in mice. Prog Neuropsychopharmacol Biol Psychiatry, 31:858-66.

Moore K. 2004. Duloxetine: a new approach for treating stress urinary incontinence? Int J Gynecol Obstet, 86:53-62.

Morilak DA, Barrera G, Echevarria DJ, et al. 2005. Role of brain norepinephrine in the behavioral response to stress. Prog Neuropsychopharmacol Biol Psychiatry, 29:1214-24.

Nemeroff CB, Schatzberg AF, Goldstein DJ, et al. 2002. Duloxetine for the treatment of major depressive disorder. Psychopharmacol Bull, 36:106-32.

Nutt DJ, Stein DJ. 2006. Understanding the neurobiology of comorbidity in anxiety disorders. CNS Spectr, 11:13-20.

Ohara K, Suzuki Y, Ochiai M, et al. 1999. A variable-number-tandemrepeat of the serotonin transporter gene and anxiety disorders. Prog Neuropsychopharmacol Biol Psychiatry, 23:55-65.

Pitsikas N. 2000. Duloxetine Eli Lilly and Co. Curr Opin Investig Drugs, $1: 116-21$.

Pollack MH. 2001. Optimizing pharmacotherapy of generalized anxiety disorder to achieve remission. J Clin Psychiatry, 62:20-5.

Pollack MH, Endicott J, Liebowitz M, et al. 2008. Examining quality of life in patients with generalized anxiety disorder: Clinical relevance and response to duloxetine treatment. J Psychiatr Res, Jan 24 [Epub ahead of print].

Rickels K, Pollack MH, Sheehan DV, et al. 2000. Efficacy of extendedrelease venlafaxine in nondepressed outpatients with generalized anxiety disorder. Am J Psychiatry, 157:968-74.

Rickels K, Pollack MH, Feltner DE, et al. 2005. Pregabalin for treatment of generalized anxiety disorder: a 4-week, multicenter, double-blind, placebo-controlled trial of pregabalin and alprazolam. Arch Gen Psychiatry, 62:1022-30.
Rynn MA, Brawman-Mintzer O. 2004. Generalized anxiety disorder: acute and chronic treatment. CNS Spectr, 9:716-23.

Rynn M, Russell J, Erickson J, et al. 2008. Efficacy and safety of duloxetine in the treatment of generalized anxiety disorder: a flexible-dose, progressivetitration, placebo-controlled trial. Depress Anxiety, 25:182-9.

Russell JM, Weisberg R, Fava M, et al. 2007. Efficacy of duloxetine in the treatment of generalized anxiety disorder in patients with clinically significant pain symptoms. Depress Anxiety, Jun 22 [Epub ahead of print].

Sharma A, Goldberg MJ, Cerimele BJ. 2000. Pharmacokinetics and safety of duloxetine, a dual-serotonin and norepinephrine reuptake inhibitor. J Clin Pharmacol, 40:161-7.

Shearer SL. 2007. Recent advances in the understanding and treatment of anxiety disorders. Prim Care, 34:475-504.

Sheehan DV, Sheehan KH. 2007. Current approaches to the pharmacologic treatment of anxiety disorders. Psychopharmacol Bull, 40:98-109.

Shelton RC, Andorn AC, Mallinckrodt CH, et al. 2007. Evidence for the efficacy of duloxetine in treating mild, moderate, and severe depression. Int Clin Psychopharmacol, 22:348-55.

Stahl SM, Grady MM, Moret C, et al. 2005. SNRIs: their pharmacology, clinical efficacy, and tolerability in comparison with other classes of antidepressants. CNS Spectr, 10:732-47.

Troelsen KB, Nielsen EO, Mirza NR. 2005. Chronic treatment with duloxetine is necessary for an anxiolytic-like response in the mouse zero maze: the role of the serotonin transporter. Psychopharmacology, 181:741-50.

Van Bockstaele EJ. 2000. Multiple substrates for serotonergic modulation of rat locus coeruleus neurons and relationships with kainate receptors. Brain Res Bull, 51:433-42.

Vaswani M, Linda FK, Ramesh S. 2003. Role of selective serotonin reuptake inhibitors in psychiatric disorders: a comprehensive review. Prog Neuropsychopharmacol Biol Psychiatry, 27:85-102.

Wernicke JF, Pritchett YL, D'Souza DN, et al. 2006. A randomized controlled trial of duloxetine in diabetic peripheral neuropathic pain. Neurology, 67:1411-20.

Westanmo AD, Gayken J, Haight R. 2005. Duloxetine: a balanced and selective norepinephrine- and serotonin-reuptake inhibitor. Am J Health Syst Pharm, 62:2481-90.

Westenberg HG, Sandner C. 2006. Tolerability and safety of fluvoxamine and other antidepressants. Int J Clin Pract, 60:482-91.

Williams VSL, Baldwin DS, Hogue SL, et al. 2006. Estimating the prevalence and impact of antidepressant-induced sexual dysfunction in 2 European countries: a cross-sectional patient survey. J Clin Psychiatry, 67:204-10.

Wang PS, Lane M, Olfson M, et al. 2005. Twelve-month use of mental health services in the United States: results from the National Comorbidity Survey Replication. Arch Gen Psychiatry, 62:629-40.

Wong DT. 1998. Duloxetine (LY248686): an inhibitor of serotonin and noradrenaline uptake and an antidepressant drug candidate. Exp Opin Investig Drugs, 7:1-9.

Wong DT, Bymaster FP. 2002. Dual serotonin and noradrenaline uptake inhibitor class of antidepressants potential for greater efficacy or just hype? Prog Drug Res, 58:169-222.

Wong DT, Bymaster FP, Mayle DA, et al. 1993. LY248686, a new inhibitor of serotonin and norepinephrine uptake. Neuropsychopharmacology, 8:23-33.

Wrase J, Reimold M, Puls I, et al. 2006. Serotonergic dysfunction: brain imaging and behavioral correlates. Cogn Affect Behav Neurosci, 6:53-61. 
